\title{
External validation of a prognostic model incorporating quantitative PET image features in oesophageal cancer
}

Citation for published version (APA):

Foley, K. G., Shi, Z., Whybra, P., Kalendralis, P., Larue, R., Berbee, M., Sosef, M. N., Parkinson, C., Staffurth, J., Crosby, T. D. L., Roberts, S. A., Dekker, A., Wee, L., \& Spezi, E. (2019). External validation of a prognostic model incorporating quantitative PET image features in oesophageal cancer. Radiotherapy and Oncology, 133, 205-212. https://doi.org/10.1016/j.radonc.2018.10.033

Document status and date:

Published: 01/04/2019

DOI:

10.1016/j.radonc.2018.10.033

Document Version:

Publisher's PDF, also known as Version of record

\section{Document license:}

Taverne

Please check the document version of this publication:

- A submitted manuscript is the version of the article upon submission and before peer-review. There can be important differences between the submitted version and the official published version of record.

People interested in the research are advised to contact the author for the final version of the publication, or visit the DOI to the publisher's website.

- The final author version and the galley proof are versions of the publication after peer review.

- The final published version features the final layout of the paper including the volume, issue and page numbers.

Link to publication

\footnotetext{
General rights rights.

- You may freely distribute the URL identifying the publication in the public portal. please follow below link for the End User Agreement:

www.umlib.nl/taverne-license

Take down policy

If you believe that this document breaches copyright please contact us at:

repository@maastrichtuniversity.nl

providing details and we will investigate your claim.
}

Copyright and moral rights for the publications made accessible in the public portal are retained by the authors and/or other copyright owners and it is a condition of accessing publications that users recognise and abide by the legal requirements associated with these

- Users may download and print one copy of any publication from the public portal for the purpose of private study or research.

- You may not further distribute the material or use it for any profit-making activity or commercial gain

If the publication is distributed under the terms of Article $25 \mathrm{fa}$ of the Dutch Copyright Act, indicated by the "Taverne" license above, 
Orignal Article

\title{
External validation of a prognostic model incorporating quantitative PET image features in oesophageal cancer
}

\author{
Kieran G. Foley ${ }^{\mathrm{a}, *}$, Zhenwei Shi ${ }^{\mathrm{b}, 1}$, Philip Whybra ${ }^{\mathrm{c}}$, Petros Kalendralis ${ }^{\mathrm{b}}$, Ruben Larue ${ }^{\mathrm{b}}$, Maaike Berbee ${ }^{\mathrm{b}}$, \\ Meindert N. Sosef ${ }^{\mathrm{d}}$, Craig Parkinson ${ }^{\mathrm{c}}$, John Staffurth ${ }^{\mathrm{a}, \mathrm{e}}$, Tom D.L. Crosby ${ }^{\mathrm{e}}$, Stuart Ashley Roberts ${ }^{\mathrm{f}}$, \\ Andre Dekker ${ }^{\mathrm{b}}$, Leonard Wee ${ }^{\mathrm{b}}$, Emiliano Spezi ${ }^{\mathrm{c}, \mathrm{d}}$

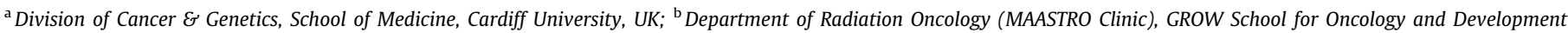

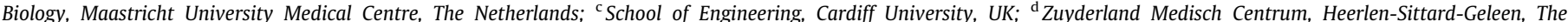 \\ Netherlands; ${ }^{\mathrm{e}}$ Velindre Cancer Centre; and ${ }^{\mathrm{f}}$ Department of Clinical Radiology, University Hospital of Wales, Cardiff, UK
}

\section{A R T I C L E I N F O}

Article history:

Received 2 May 2018

Received in revised form 23 October 2018

Accepted 25 October 2018

Available online 10 November 2018

\section{Keywords:}

Oesophageal cancer

Positron-emission tomography

Radiomics

Survival

Prognosis

\begin{abstract}
A B S T R A C T
Aim: Enhanced prognostic models are required to improve risk stratification of patients with oesophageal cancer so treatment decisions can be optimised. The primary aim was to externally validate a published prognostic model incorporating PET image features. Transferability of the model was compared using only clinical variables.

Methods: This was a Transparent Reporting of a multivariate prediction model for Individual Prognosis Or Diagnosis (TRIPOD) type 3 study. The model was validated against patients treated with neoadjuvant chemoradiotherapy according to the Neoadjuvant chemoradiotherapy plus surgery versus surgery alone for oesophageal or junctional cancer (CROSS) trial regimen using pre- and post-harmonised image features. The Kaplan-Meier method with log-rank significance tests assessed risk strata discrimination. A Cox proportional hazards model assessed model calibration. Primary outcome was overall survival (OS). Results: Between 2010 and 2015, 449 patients were included in the development $(n=302)$, internal validation $(n=101)$ and external validation $(n=46)$ cohorts. No statistically significant difference in OS between patient quartiles was demonstrated in prognostic models incorporating PET image features $\left(X^{2}=1.42, \mathrm{df}=3, p=0.70\right)$ or exclusively clinical variables (age, disease stage and treatment; $X^{2}=1.19$, $\mathrm{df}=3, p=0.75$ ). The calibration slope $\beta$ of both models was not significantly different from unity $(p=0.29$ and 0.29 , respectively). Risk groups defined using only clinical variables suggested differences in OS, although these were not statistically significant $\left(X^{2}=0.71, \mathrm{df}=2, p=0.70\right)$.

Conclusion: The prognostic model did not enable significant discrimination between the validation risk groups, but a second model with exclusively clinical variables suggested some transferable prognostic ability. PET harmonisation did not significantly change the results of model validation.

Crown Copyright $\odot 2018$ Published by Elsevier B.V. All rights reserved. Radiotherapy and Oncology 133
\end{abstract}

(2019) 205-212
The prognosis of patients with oesophageal cancer is poor with overall 5-year survival approximately 15\% [1]. Oesophageal cancer

Abbreviations: LNMs, lymph node metastases; PET, positron-emission tomography; NACRT, neo-adjuvant chemoradiotherapy; CROSS, neoadjuvant chemoradiotherapy plus surgery versus surgery alone for oesophageal or junctional cancer; TRIPOD, Transparent Reporting of a multivariate prediction model for Individual Prognosis Or Diagnosis; GI, gastrointestinal; MDT, multi-disciplinary team; CaNISC, Cancer Network Information System; ATLAAS, Automatic Tree-based Learning Algorithm for Advanced Segmentation; CI, confidence interval; TLG, tumour lesion glycolysis; OS, overall survival; IBSI, Image Biomarker Standardisation Initiative.

* Corresponding author at: Division of Cancer \& Genetics, School of Medicine, Cardiff University, CF14 4XN, UK.

E-mail address: foleykg@cardiff.ac.uk (K.G. Foley).

${ }^{1}$ Equal contribution. is the eighth most common malignancy worldwide, accounting for around 400,000 deaths each year [2].

Treatment strategies of patients with oesophageal cancer are currently informed by radiological staging. Accurate staging is vital to inform clinicians of the likely prognosis of each patient and to appropriately risk stratify patients, ensuring the best individual management plan is decided upon. However, the stagnation in survival rate over recent decades suggests that staging accuracy, treatment selection and prognosis could be much improved. For example, lymph node metastases (LNMs) are one of the major prognostic indicators in oesophageal cancer, but there is evidence that regional lymph node staging ( $N$-stage) is presently suboptimal $[3,4]$. Therefore, enhanced staging methods are required to 
improve prognostication and subsequent risk stratification of patients.

Oesophageal cancer is typically confirmed by a small-sample biopsy taken during endoscopic examination. Despite advances in genomics, no molecular prognostic markers are currently in routine clinical use [5]. It has been proposed that additional tumour phenotype information may be derived by quantitative analysis of Positron Emission Tomography (PET) scans [6]. "Radiomics" broadly refers to automated, computerised and high-throughput extraction of quantitative image markers (features) from a large corpus of radiological images [7]. Radiomic features typically include histogram metrics (e.g. mean and maximum), shape descriptors (e.g. longest axis length and compactness) and textures (e.g. continuous length of voxels with similar intensities) [8]. These features can be sensitive to differences in image parameters such as slice thickness [9]. Post-reconstruction harmonisation methods have been proposed to adjust for these differences, thus promoting standardised research between centres [10].

The primary aim of this study was to test the generalisability of a UK single-centre oesophageal cancer prognostic model incorporating radiomic features [11] firstly pre-harmonisation, then post-harmonisation, against a cohort of oesophageal cancer patients treated exclusively with neo-adjuvant chemoradiotherapy (NACRT) according to the Dutch NACRT plus surgery versus surgery alone for oesophageal/junctional cancer (CROSS) trial regimen [12]. A widely generalisable prognostic model incorporating radiomic features of primary tumours might offer clinicians complimentary data beyond traditional prognostic factors that will assist treatment decision making and risk stratification $[11,13]$. The secondary aim was to compare prognostic models with and without PET image features between cohorts to provide further validation.

\section{Materials and methods}

This study was designed as a Transparent Reporting of a multivariate prediction model for Individual Prognosis Or Diagnosis (TRIPOD) type 3 external independent validation study [14]. A previously published prognostic model had been developed and internally validated in patients with oesophageal cancer. Details of model development have been provided in Foley et al. [11]. Briefly, the prognostic model had only been evaluated by same-centre internal validation in patients managed by the South-East Wales Regional Upper Gastrointestinal (GI) Cancer Multi-Disciplinary Team (MDT), United Kingdom. A suitable independent cohort was not accessible at the time of publication. Institutional board review (IRB) approval was granted for the development of the prognostic model (REF 14/WA/1208). The prognostic model was developed as part of a larger study investigating the prognostic significance of image texture analysis in gastro-oesophageal cancer (STAGE), and from here-on will be known as the STAGE cohort. The external validation cohort comprised patients treated with the CROSS regimen in The Netherlands. IRB permission was obtained for the external validation cohort.

\section{Patient cohorts}

In total, 449 patients were included in the development and validation of this prognostic model. Fig. 1 details the number of patients in each cohort and the reasons for exclusion of patients from the CROSS validation cohort. The largest number of patient exclusions $(n=23)$ from the CROSS cohort were because of the pre-defined metabolic tumour volumes (MTV) adopted in Foley et al [11] and used in this current study for consistency. A sensitivity analysis of these excluded cases has been included in Appendix
B. Other main reasons for patient exclusion were different calibration units $(n=11)$ and ATLAAS segmentation failure $(n=7)$.

\section{Primary outcome}

The primary endpoint of the published prognostic model is overall survival, defined as the number of months survived after the date of diagnosis until death or last day of follow-up. Dates of death were obtained from the Cancer National Information System Cymru (CaNISC) database (Velindre NHS Trust, Wales), reported by the Office for National Statistics. Dates of death of patients in the CROSS cohort were obtained from the national registry. In both cohorts, local researchers were not blinded to the dates of death. A uniform and standardised procedure for autosegmentation and radiomic computation was implemented at each centre to ensure consistent methodology.

\section{Tumour segmentation}

Primary tumours were segmented on PET images using an automatic tree-based learning algorithm for advanced segmentation (ATLAAS) [15]. The benefit of ATLAAS is that inter-observer variability in contouring is eliminated. Full details regarding the use of ATLAAS in this study are provided in Foley et al. and Berthon et al. $[11,15]$.

The following model equation (Eq. (1)) was used to calculate a prognostic score for each patient. This equation was derived using published methods [16].

Prognostic score $=$ Stage Group $* 0.397$ - Treatment $* 1.094+$ Age $* 0.024-$ $\log ($ Histogram Energy $) * 1.320+\log ($ TLG $) * 1.748+$ Histogram Kurtosis $* 0.198$

\section{External validation}

The ATLAAS code and equations to calculate each of the PET image features were shared between institutions. The primary tumours on the PET scans of the CROSS patients were then segmented using ATLAAS and the MTVs produced were visually assessed for adequacy for quality control. Validation was firstly performed with pre-harmonisation metrics and then repeated with post-harmonisation PET features to adjust for potential differences between scanners. Fully anonymised data were then shared between institutions.

Different PET/CT scanners and protocols were used across the cohorts (Appendix A). Radiomic features are known to change significantly as a function of scanner model, image acquisition or reconstruction settings, therefore we explored using the postreconstruction Combat harmonisation method [17] to harmonise features extracted from images acquired across different scanners. Slice thickness was chosen for harmonisation because images from one scanner had different thickness values, which resulted in 5 categories (Appendix A, Table A.1). Further details of the cohorts, treatments received, PET/CT protocols, metric equations, variation in image features and the post-reconstruction PET harmonisation Combat method [17], used to adjust for batch effects across different datasets, have been provided in Appendix A.

\section{Statistical analysis}

Categorical data are described as frequency (percent) and continuous variables as median (range) and differences assessed with appropriate non-parametric tests. There was no missing data in the development cohort and cases with missing data were excluded from the validation CROSS cohort. Patient characteristics at staging were compared for each cohort. Boxplots were generated locally on 


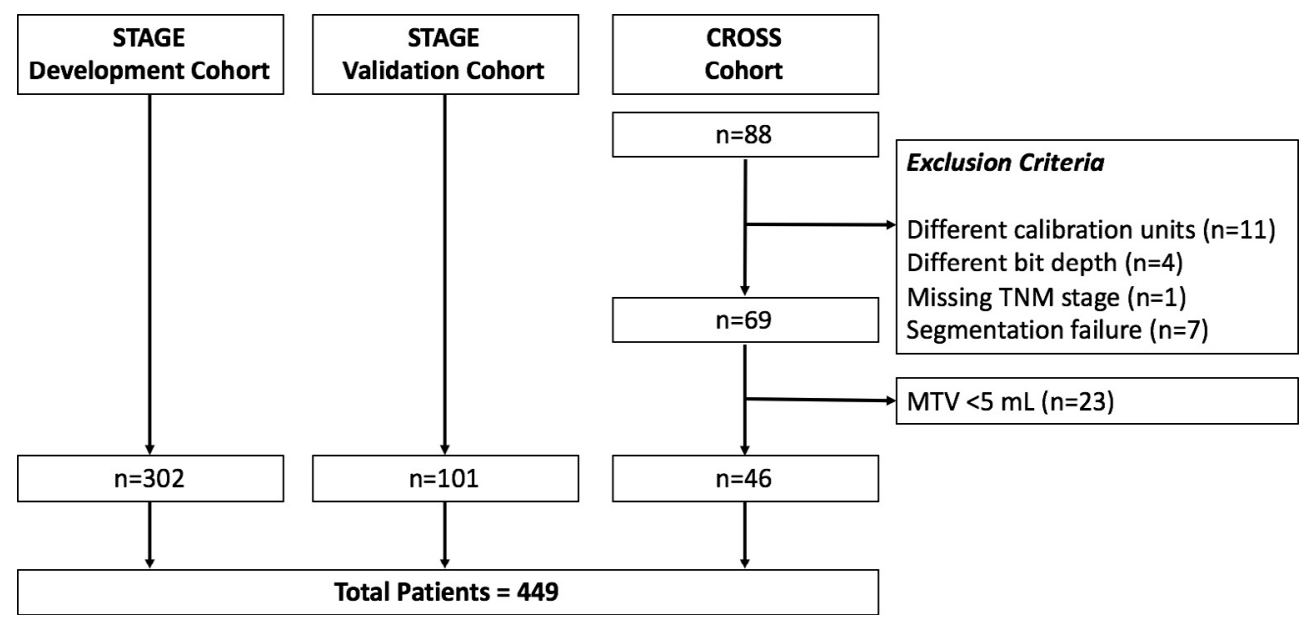

Fig. 1. Study flowchart describing the numbers of patients in each cohort and reasons for exclusions from the CROSS cohort.

each cohort to compare the distributions of the model variables. Firstly, the published model was applied to 46 suitable patients in the CROSS cohort prior to PET harmonisation. A second model validation was then performed using image features calculated postharmonisation. Model discrimination was evaluated using the log-rank test; a $p$-value of $<0.05$ was defined as statistically significant. Model calibration followed a standard test procedure detailed in [18], and which has been previously implemented in [19]. In this study, we define model discrimination as preserved if the $p$-value of the calibration slope $\beta=1$ is $>0.05$. Thirdly, we performed the same validation steps for a prognostic model developed on the same STAGE cohort, but exclusively using clinical variables (age at diagnosis, stage and treatment) and no imaging based variables. Statistical analysis was performed with SPSS version 23.0 (IBM, Chicago, USA) and MATLAB version 9.0 (MathWorks, Natick, MA).

\section{Results}

The baseline characteristics of the STAGE development, validation and CROSS cohorts are detailed in Table 1. The median overall survival of the CROSS cohort was 25 months (95\% confidence inter$\operatorname{val}(\mathrm{CI}) 23.0-31.4)$. The median overall survival of the STAGE development and validation cohorts was 16.0 months (95\% CI 13.818.2 ) and 14.0 months (95\% CI 10.4-17.6), respectively.

Boxplots were constructed to compare the values of $\log (\mathrm{TLG})$, $\log$ (Histogram Energy) and Histogram Kurtosis in between the STAGE and CROSS cohorts (Fig. 2). Additional boxplots and descriptive statistics of PET feature values pre- and post-harmonisation are included in Appendix B. There were similar mean values and distributions of the 3 variables between STAGE and CROSS cohorts, although a greater number of outliers were observed for Histogram Kurtosis in the STAGE cohort. This is probably due to a larger number of patients and greater range in MTV of the primary tumours included in the STAGE cohort. (Table B.1).

A prognostic model containing clinical variables only was calculated from the STAGE development cohort using identical data from the original study. Age at diagnosis (HR 1.025, 95\% CI $1.011-1.040, p<0.001)$, stage $(0.337,0.243-0.468, p<0.001)$ and treatment $(1.462,1.187-1.802, p<0.001)$ were all independently and significantly associated with overall survival.

\section{Prognostic model developed by clinical and radiomic features}

\section{Pre-harmonisation}

Kaplan-Meier analysis did not demonstrate a significant difference in overall survival between patient quartiles in the CROSS cohort $\left(X^{2}=1.27, \mathrm{df}=3, p=0.74\right)$ (Fig. 3 ). The HRs of quartiles 2 , 3 and 4 compared to quartile 1 was 0.89 (95\% CI 0.29-2.75), 1.36 (95\% CI $0.47-3.92)$ and 0.78 (95\% CI $0.25-2.41)$, respectively. The calibration slope $\beta$ of the prognostic score in the CROSS cohort was 1.09 (standard error (SE) 0.41). $\beta$ is not significantly different from $1(p=0.84)$, which indicates that model discrimination is preserved.

The mean overall survival for patient quartiles 1-4 were 34.0 months (95\% CI 19.0-49.2), 29.5 months (95\% CI 19.5-39.5), 25.9 months (95\% CI 14.8-37.0) and 41.2 months (95\% CI 25.956.4 ), respectively. Median overall survival could not be calculated for all quartiles. The median prognostic score for quartiles $1-4$ was $-0.51(n=11$, range -1.14 to -0.37$),-0.15(n=11$, range -0.36 to $0.01), 0.20(n=11$, range $0.04-0.30)$ and $0.48(n=13$, range $0.30-$ $1.16)$, respectively.

\section{Post-harmonisation}

Following post-reconstruction PET harmonisation, repeated Kaplan-Meier analysis did not demonstrate a significant difference in overall survival between patient quartiles in the CROSS cohort $\left(X^{2}=1.42, \mathrm{df}=3, p=0.70\right)$. (Fig. 3 ) The HRs of quartiles 2,3 and 4 compared to quartile 1 was 0.78 (95\% CI $0.24-2.55), 1.47$ (95\% CI $0.50-4.25)$ and 1.15 (95\% CI 0.39-3.40), respectively. The calibration slope $\beta$ of the prognostic score in the CROSS cohort was 1.26 (standard error (SE) 0.22). $\beta$ is not significantly different from 1 $(p=0.29)$, which indicates that model discrimination is preserved. The adjusted survival data for the patient quartiles are available in Appendix B.

These results indicate that PET harmonisation did not have a substantial effect on model validation, with similar results obtained using both methods.

\section{Prognostic model developed with clinical features only}

The median prognostic score of the model developed with clinical variables only was -2.68 (range -4.89 to -0.17 ). As shown in Fig. 4, Kaplan-Meier analysis did not demonstrate a significant difference in overall survival between patient quartiles in the CROSS cohort $\left(X^{2}=1.19, \mathrm{df}=3, p=0.75\right)$. The HRs of quartiles 2,3 and 4 compared to quartile 1 was 0.93 (95\% CI $0.27-3.23), 1.41$ (95\% CI $0.45-4.43$ ) and 1.53 (95\% CI 0.51-4.57), respectively. The calibration slope $\beta$ of the prognostic score in the CROSS cohort was 2.15 (SE 0.72). $\beta$ is not significantly different from $1(p=0.29)$, which indicates that model discrimination is preserved.

In the prognostic model with clinical variables only, patients in quartiles 2 \& 3 were combined to create an intermediate risk group, following a previously published method [20] (Fig. 5). 
Table 1

Baseline characteristics of patients in development, validation and CROSS cohorts.

\begin{tabular}{|c|c|c|c|c|}
\hline Frequency (\%) & STAGE development cohort $(n=302)$ & STAGE validation cohort $(n=101)$ & CROSS cohort $(n=46)$ & $p$-Value \\
\hline Median Age & $\begin{array}{l}67.0 \text { years } \\
\text { (Range 39-83) }\end{array}$ & $\begin{array}{l}69.0 \text { years } \\
\text { (Range } 39-84 \text { ) }\end{array}$ & $\begin{array}{l}64.5 \text { years } \\
\text { (Range } 47-77.8 \text { ) }\end{array}$ & 0.114 \\
\hline Gender (M: F) & 227 (75.2): $75(24.8)$ & $78(77.2): 23(22.8)$ & 38 (82.6): $8(17.4)$ & 0.528 \\
\hline \multicolumn{5}{|l|}{ Histology } \\
\hline Adeno & $237(78.5)$ & $79(78.2)$ & $39(84.8)$ & \\
\hline SCC & $65(21.5)$ & $22(21.8)$ & $7(15.2)$ & 0.602 \\
\hline \multicolumn{5}{|l|}{ Tumour location } \\
\hline Oesophagus & $192(63.6)$ & $47(46.5)$ & $28(60.9)$ & 0.010 \\
\hline Gastro-oesophageal junction & $110(36.4)$ & $54(53.5)$ & $18(39.1)$ & \\
\hline \multicolumn{5}{|l|}{ Stage groups } \\
\hline Stage 1 & $17(5.6)$ & $2(2.0)$ & $2(4.4)$ & 0.018 \\
\hline Stage 2 & $56(18.5)$ & $24(23.8)$ & $10(21.7)$ & \\
\hline Stage 3 & $160(53.1)$ & $57(56.4)$ & $33(71.7)$ & \\
\hline Stage 4 & $69(22.8)$ & $18(17.8)$ & $1(2.2)$ & \\
\hline Treatment & & & & $<0.001$ \\
\hline Curative & $158(52.3)$ & $50(49.5)$ & $46(100)$ & \\
\hline SA & $24(15.2)$ & $4(8.0)$ & $0(0.0)$ & \\
\hline NACT & $67(42.4)$ & $23(46.0)$ & $0(0.0)$ & \\
\hline NACRT & $13(8.2)$ & $7(14.0)$ & $46(100)$ & \\
\hline dCRT & $54(34.2)$ & $16(32.0)$ & $0(0.0)$ & \\
\hline Palliative & $144(47.7)$ & $51(50.5)$ & $0(0.0)$ & \\
\hline Overall survival & & & & $<0.001$ \\
\hline Alive & $70(23.2)$ & $43(42.6)$ & $20(43.5 \%)$ & \\
\hline Dead & $232(76.8)$ & $58(57.4)$ & $26(51.5 \%)$ & \\
\hline
\end{tabular}

SCC squamous cell carcinoma; SA surgery alone; NACT neo-adjuvant chemotherapy; NACRT neo-adjuvant chemoradiotherapy; dCRT definitive chemoradiotherapy. Chi-square test

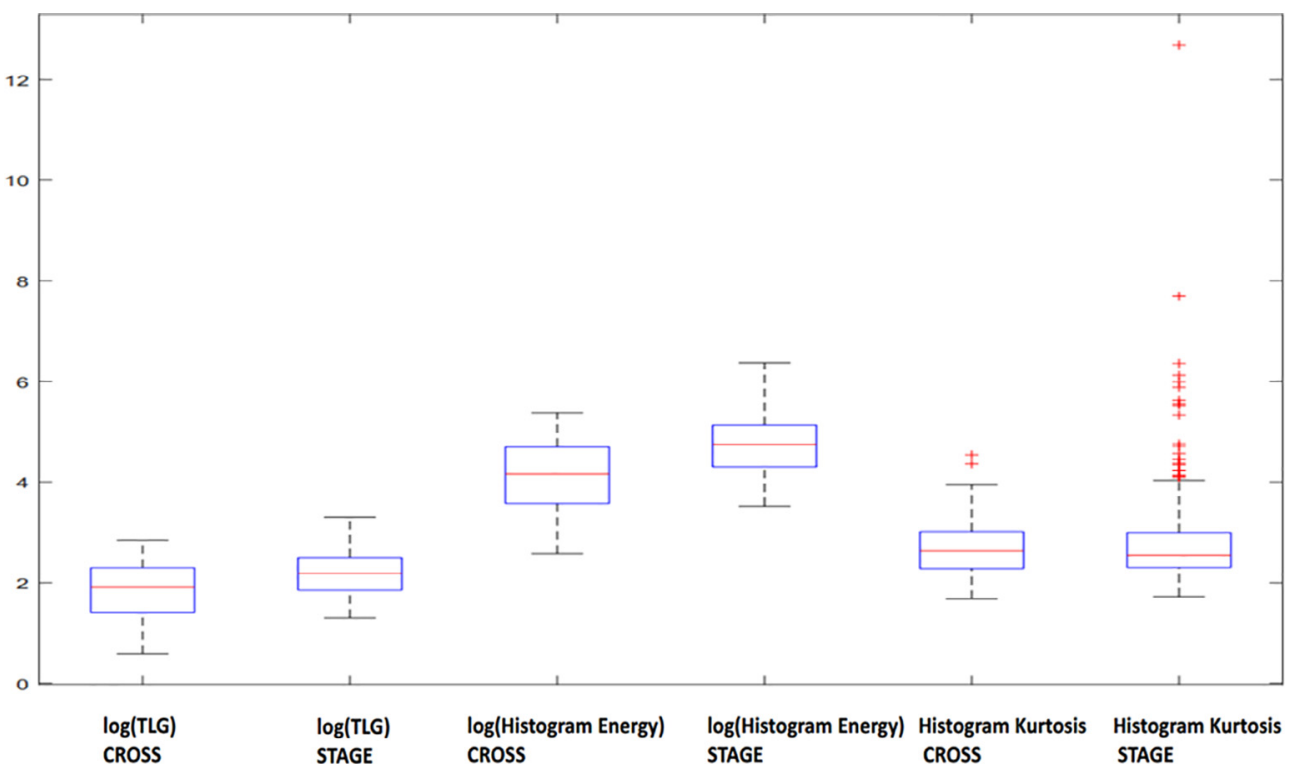

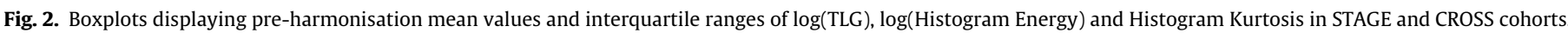

Applying Bonferroni correction, there was no statistically significance difference between the low, intermediate and high risk groups $\left(X^{2} 0.712\right.$, df $\left.2, p=0.701\right)$ but a separation in overall survival curves was observed (intermediate risk vs low risk HR 1.16 (95\% CI 0.41-3.30 and high risk vs low risk HR 1.53 (95\% CI $0.51-4.58)$ ). The calibration slope $\beta=2.15$ (SE 0.72, $p$-value 0.29 ) indicating model discrimination was preserved.

\section{Discussion}

Patients with oesophageal cancer have a poor prognosis and the incidence of the disease is increasing [21]. Despite advances in modern healthcare, survival rates remain low. Enhanced staging algorithms are required to improve the accuracy of staging, which informs clinicians of the likely prognosis and provides subsequent patient risk stratification. Prognostic models incorporating radiomic features are one strategy being investigated for this purpose.

This external validation study has shown that results of a developed prognostic model combining clinical risk factors and PET radiomic features was not replicated in a cohort of patients treated with the CROSS trial regimen. However, when a prognostic model including only clinical variables from the STAGE development cohort was tested, some aspects of the model were indicative of transferability to the CROSS cohort. Our data show that clinical features of oesophageal cancer remain prognostic across different countries and studies. 


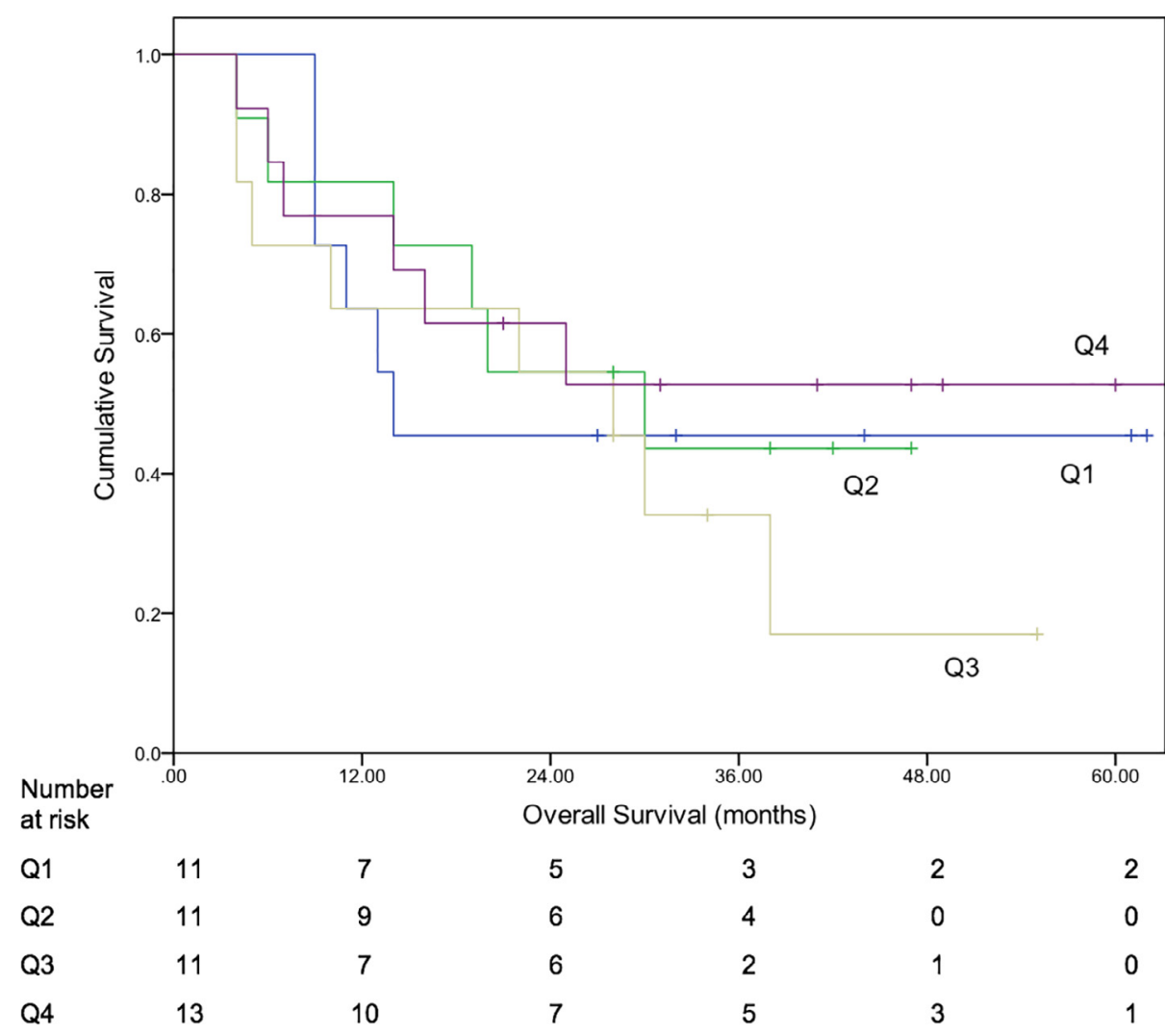

Fig. 3. Cumulative survival curves of patient quartiles $(\mathrm{Q} 1-4)$ in CROSS cohort using model developed with clinical and radiomic features $\left(X^{2}=1.27\right.$, $\left.\mathrm{df}=3, p=0.74\right)$.

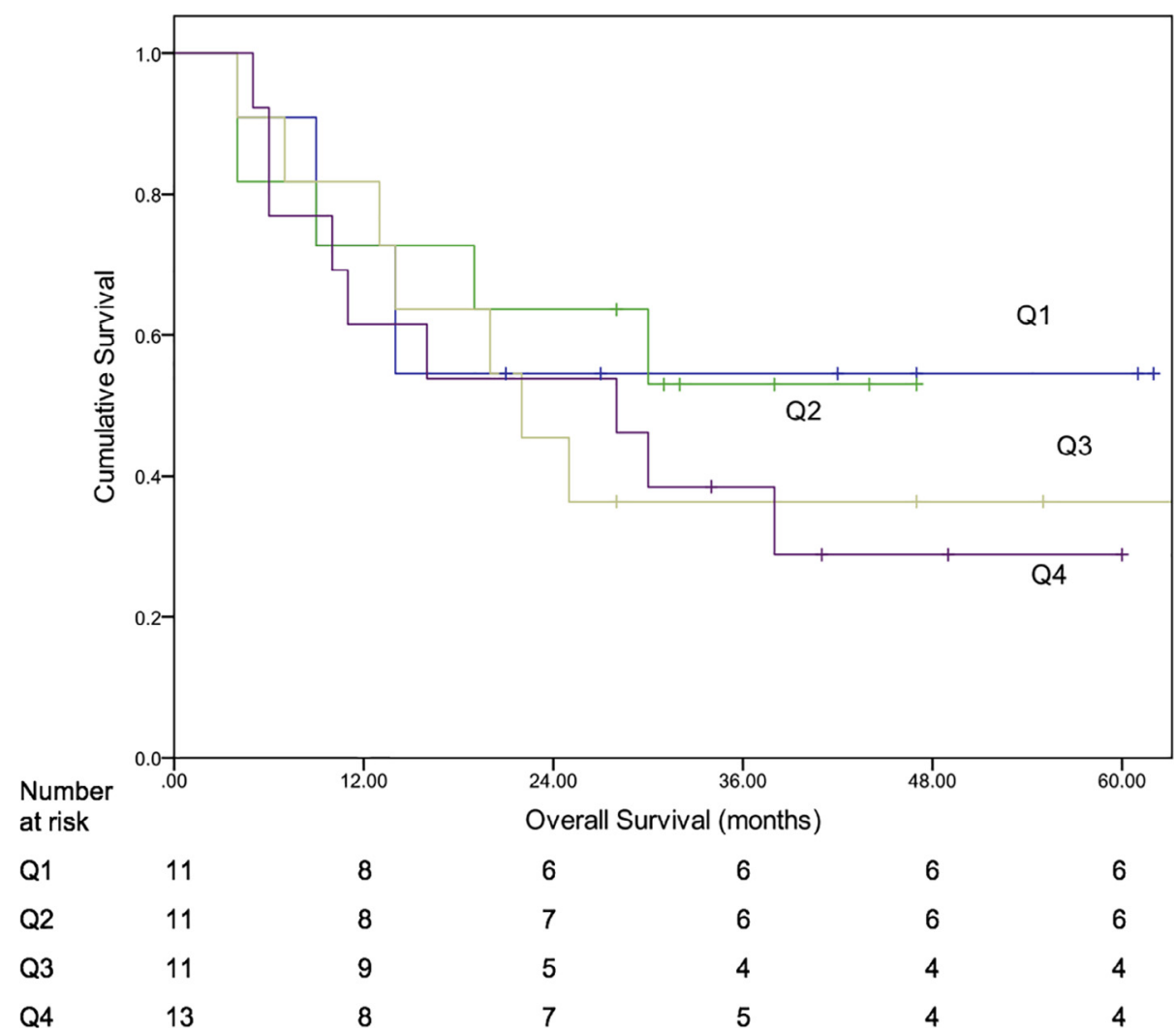

Fig. 4. Cumulative survival curves of patient quartiles $(\mathrm{Q} 1-4)$ in CROSS cohort using model developed with clinical features only $\left(X^{2}=1.19\right.$, $\left.\mathrm{df}=3, p=0.75\right)$. 


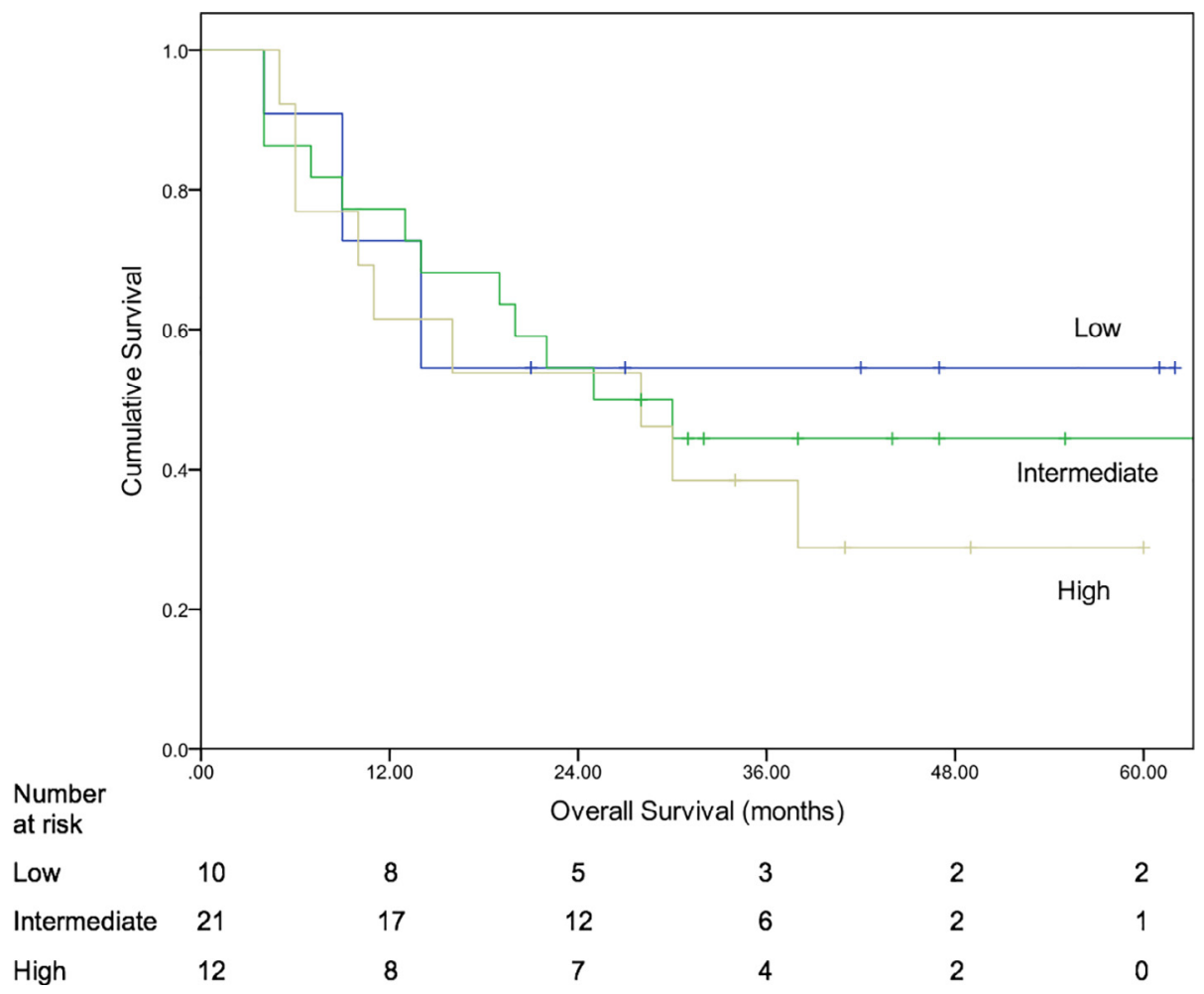

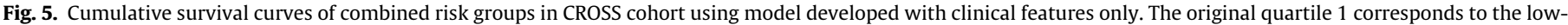
risk group, quartiles $2 \& 3$ were combined to create an intermediate risk group and quartile 4 corresponds to the high-risk group.

Despite not being able to replicate the validation results of the published prognostic model, this study remains clinically important because more accurate staging of oesophageal cancer is essential to improve survival rates. Validated prognostic and predictive radiomic models are one strategy to improve radiological staging of oesophageal cancer [22]. Greater staging accuracy will improve patient risk stratification, which is critically important for optimising personalised treatment decision-making. Once validated, staging algorithms incorporating radiomics may enable clinicians to decide upon the best management plan from the outset of diagnosis, therefore providing the greatest chance of survival for each patient.

A number of important methodological reasons in the modelling process may have contributed to the lack of external validity of the prognostic model when transported to the CROSS observations. First, the PET image acquisition protocols in the CROSS regimen cohort may not have been as strictly policed as in the STAGE study, leading to divergence in PET acquisition parameters. (Table A.1) All patients in STAGE $(n=403)$ were staged using the same PET/CT scanner and protocol. However, different PET/CT scanners and protocols were used in both the STAGE and CROSS cohorts.

Harmonising PET image features demonstrated little improvement in the model validity between cohorts, which supports this post-reconstruction method in external validation radiomic studies and suggests that harmonisation had little influence. These findings contradict those of Orlhac et al. [10]. Several factors could explain the lack of effect. The clinical variables of patient age, TNM stage and treatment are likely to have the greatest impact on overall survival compared to the image features. The PET features used in the original model by Foley et al (TLG, Histogram Energy and Histogram Kurtosis) were not investigated in Orlhac et al. Furthermore, although the Combat algorithm has been used in genomics, it has not yet been validated in radiomics. A consensus on uniformly standardised PET imaging protocols is required for multiinstitutional validation of prognostic/predictive models incorporating radiomics. [23]

Second, the prognostic model excluded patients with small MTV $<5 \mathrm{~mL}$, thus further reducing the number of CROSS patients that were eligible for validation. The small patient numbers in the external validation cohort limits the ability to replicate the results of the STAGE prognostic model. This study is likely to be under-powered and improved validation could be achieved by increasing the cohort size. Patients with a smaller MTV were more likely to be suitable for radical therapy and therefore eligible for recruitment into the CROSS trial. When the excluded small MTV cases were tested in the sensitivity analysis included in Appendix $B$, no significant difference in overall survival between patient quartiles remained $\left(X^{2}=3.85, \mathrm{df}=3, p=0.28\right)$. In addition, evidence at the time of prognostic model development suggested possible unstable segmentation at smaller MTVs and an increase in redundant (highly cross-correlated) radiomic data that can be extracted. [24] There is no clear consensus on minimum MTV in PET radiomic studies. One study recommends excluding MTVs of $<45 \mathrm{~mL}$, although only one calculation choice for local entropy was evaluated in this study, despite the many possibilities of discretisation steps and matrices available [25]. Other studies have suggested excluding patients with a primary MTV of $<10 \mathrm{~mL}$ $[26,27]$. Despite this, prognostic models including image features extracted from small tumour volumes can still be developed [8]. The original model by Foley et al. did not examine a wide range of higher order features, some of which may have turned out to be reproducible and prognostically significant with the expanded dataset. However, since the scope of this study was only the feasible generalisability of the original model, we did not re-analyse using additional textural features. 
Third, the development of the previous prognostic model did not include an exhaustive radiomic feature selection step to identify features that would be robustly reproducible within the STAGE cohort and hence more likely to be transferable to the CROSS cohort [8]. Details of the PET variables implemented in the developed prognostic model can be found in Foley et al. [11]. These variables were shown to have prognostic significance in the early radiomic literature [28-30] and were implemented identically.

More studies are required to test the reliability, robustness and additional value of PET image features across a range of MTVs and between different PET/CT scanners $[9,26]$. Regarding the original model, TLG and Histogram Energy have shown good reproducibility results, however there is mixed evidence for Histogram Kurtosis [31]. Previous studies have found significant associations between higher order features and overall survival [29] and that the amount of complementary radiomic information gained increases with larger MTVs [26]. Despite this, the original development study did not demonstrate prognostic significance of any higher order features, although only 3 such features were investigated.

Advanced correction algorithms are being developed to harmonise features extracted from scans with different acquisition parameters, which could greatly benefit multi-centre radiomic studies and reduce variation in metrics [32].

Standardisation efforts such as the Image Biomarker Standardisation Initiative (IBSI) [33] are an important methodological step towards reducing sensitivity of radiomic features to computation (image extraction) software. Deployment of the same autosegmentation tool (ATLAAS [15]) reduced inter-observer variability in contouring and the same feature extraction software that was executed locally was used in both participating centres. These techniques are examples of standardised processes that improve the robustness of radiomic features.

Lastly, a relatively small proportion of the STAGE cohort received NACRT or surgery alone (Table 1 ). These differences may not have been adjusted for completely by the original model multivariate regression. The STAGE cohort is relatively heterogeneous cohort of patients compared to the CROSS cohort, because it was collected during an observational cohort study recruiting all patients with oesophageal cancer. Patients in the CROSS cohort were all treated with NACRT, so they share more similar characteristics. Differences between validation cohorts are important in external validation studies because the generalisation of the model can be tested at its extremes. Furthermore, this points the way forward to improved (reproducible) feature selection methodology and updating of the original model to address a more generalised clinical question.

All prognostic models must be validated in an independent external cohort before being considered for use in clinical practice because many models present optimistic and over-fitted results from development cohorts [34]. However, external validation studies are rarely performed. A review of the performance of prognostic models showed that $11 \%$ are externally validated [35]. This may explain why few developed prognostic models are adopted into clinical practice [36]. Our collaborative research group is planning to update this prognostic model and perform a further external validation study with more robust feature selection and standardised feature extraction algorithms using all tumour volumes.

In conclusion, this initial TRIPOD type 3 external validation study evaluated a prognostic model developed in oesophageal cancer patients staged with PET/CT. The prognostic model did not enable significant discrimination between patient risk groups in the CROSS cohort, but a second model including clinical variables only (age, disease stage and treatment) demonstrated transferable prognostic factors between international cohorts.

\section{Acknowledgements}

The authors wish to acknowledge the contributions of Professor Robert K Hills who developed the original prognostic model, Professor Wyn G Lewis who helped with the STAGE cohort data collection, Professor Christopher Marshall (Director of the PositronEmission Tomography Imaging Centre (PETIC) in Cardiff and members of the South-East Wales Upper GI Cancer MDT committee.

\section{Ethical statement}

Institutional review board approval was obtained.

\section{Data availability}

The data that have been used in this study are confidential and cannot be shared.

\section{Funding}

The study was partially funded by a UK Tenovus Cancer Care Grant (TIG2016/04).

\section{Competing interests}

The authors declare that they have no competing interests.

\section{Author contributions}

$\mathrm{KF}, \mathrm{AR}, \mathrm{LW}$ and ES conceived and designed the study. RL, MB, MS, PK and TC collected the data. ZS, PW, CP and PK preformed the data analysis. KF, LW, JS, TC and AD drafted the manuscript. All authors read and approved the final manuscript.

\section{Appendix A. Supplementary data}

Supplementary data to this article can be found online at https://doi.org/10.1016/j.radonc.2018.10.033.

\section{References}

[1] Cancer Research UK. Oesophageal Cancer Statistics. 2016 [Accessed November 22nd 2016]; Available from: http://www.cancerresearchuk.org/healthprofessional/cancer-statistics/statistics-by-cancer-type/oesophageal-cancer.

[2] Ferlay I, Soerjomataram I, Dikshit R, Eser S, Mathers C, Rebelo M, et al. Cancer incidence and mortality worldwide: sources, methods and major patterns in GLOBOCAN 2012. Int J Cancer 2015;136:E359-86.

[3] Kayani B, Zacharakis E, Ahmed K, Hanna GB. Lymph node metastases and prognosis in oesophageal carcinoma-a systematic review. Eur J Surg Oncol 2011;37:747-53.

[4] Foley KG, Christian A, Fielding P, Lewis WG, Roberts SA. Accuracy of contemporary oesophageal cancer lymph node staging with radiologicalpathological correlation. Clin Radiol 2017;72:e691-7.

[5] McCormick Matthews LH, Noble F, Tod J, Jaynes E, Harris S, Primrose JN, et al. Systematic review and meta-analysis of immunohistochemical prognostic biomarkers in resected oesophageal adenocarcinoma. $\mathrm{Br} J$ Cancer 2015;113:107-18.

[6] Cook GJR, Siddique M, Taylor BP, Yip C, Chicklore S, Goh V. Radiomics in PET: principles and applications. Clin Transl Imaging 2014:2:269-76.

[7] Lambin P, Rios-Velazquez E, Leijenaar R, Carvalho S, van Stiphout RG, Granton $\mathrm{P}$, et al. Radiomics: extracting more information from medical images using advanced feature analysis. Eur J Cancer 2012;48:441-6.

[8] Aerts HJ, Velazquez ER, Leijenaar RT, Parmar C, Grossmann P, Cavalho S, et al. Decoding tumour phenotype by noninvasive imaging using a quantitative radiomics approach. Nat Commun 2014;5:4006.

[9] Desseroit MC, Tixier F, Weber WA, Siegel, BA,Cheze, Le, Rest, C, Visvikis, D, et al. Reliability of PET/CT shape and heterogeneity features in functional and morphologic components of non-small cell lung cancer tumors: a repeatability analysis in a prospective multicenter cohort. J Nucl Med 2017:58:406-11.

[10] Orlhac F, Boughdad S, Philippe C, Stalla-Bourdillon H, Nioche C, Champion L, et al. A post-reconstruction harmonization method for multicenter radiomic studies in PET. J Nucl Med 2018;59:1321-8. 
[11] Foley KG, Hills RK, Berthon B, Marshall C, Parkinson C, Lewis WG, et al. Development and validation of a prognostic model incorporating texture analysis derived from standardised segmentation of PET in patients with oesophageal cancer. Eur Radiol 2018;28:428-36.

[12] van Hagen P, Hulshof MCCM, van Lanschot JJB, Steyerberg EW, van Berge Henegouwen MI, Wijnhoven BPL, et al. Preoperative chemoradiotherapy for esophageal or junctional cancer. N Engl J Med 2012;366:2074-84.

[13] Tan X, Ma Z, Yan L, Ye W, Liu Z, Liang C. Radiomics nomogram outperforms size criteria in discriminating lymph node metastasis in resectable esophageal squamous cell carcinoma. Eur Radiol 2018.

[14] Collins GS, Reitsma JB, Altman DG, Moons KG. Transparent reporting of a multivariable prediction model for individual prognosis or diagnosis (TRIPOD): the TRIPOD statement. BMJ 2015;350:g7594.

[15] Berthon B, Marshall C, Evans M, Spezi E. ATLAAS: an automatic decision treebased learning algorithm for advanced image segmentation in positron emission tomography. Phys Med Biol 2016;61:4855-69.

[16] Moons KG, Kengne AP, Woodward M, Royston P, Vergouwe Y, Altman DG, et al. Risk prediction models: I. Development, internal validation, and assessing the incremental value of a new (bio)marker. Heart 2012;98:683-90.

[17] Johnson WE, Li C, Rabinovic A. Adjusting batch effects in microarray expression data using empirical Bayes methods. Biostatistics 2007;8: $118-27$.

[18] Royston P, Altman DG. External validation of a Cox prognostic model: principles and methods. BMC Med Res Methodol 2013;13:33.

[19] Leijenaar RT, Carvalho S, Hoebers FJ, Aerts HJ, van Elmpt WJ, Huang SH, et al. External validation of a prognostic CT-based radiomic signature in oropharyngeal squamous cell carcinoma. Acta Oncol 2015;54:1423-9.

[20] Dekker A, Vinod S, Holloway L, Oberije C, George A, Goozee G, et al. Rapid learning in practice: a lung cancer survival decision support system in routine patient care data. Radiother Oncol 2014;113:47-53.

[21] Cancer Research UK. Oesophageal Cancer Incidence Statistics. 2016 [Accessed December 20th 2016]; Available from: http://www.cancerresearchuk.org/ health-professional/cancer-statistics/statistics-by-cancer-type/oesophagealcancer/incidence.

[22] van Rossum PS, Xu C, Fried DV, Goense L, Court LE, Lin SH. The emerging field of radiomics in esophageal cancer: current evidence and future potential. Transl Cancer Res 2016:5:410-23.

[23] Hatt M, Lee JA, Schmidtlein CR, Naqa IE, Caldwell C, De Bernardi E, et al. Classification and evaluation strategies of auto-segmentation approaches for PET: Report of AAPM task group No. 211. Med Phys 2017;44:e1-e42.
24] Wu W, Parmar C, Grossmann P, Quackenbush J, Lambin P, Bussink J, et al Exploratory study to identify radiomics classifiers for lung cancer histology. Front Oncol 2016;6:71.

[25] Brooks FJ, Grigsby PW. The effect of small tumor volumes on studies of intratumoral heterogeneity of tracer uptake. J Nucl Med 2014;55:37-42.

[26] Hatt M, Majdoub M, Vallieres M, Tixier F, Le Rest CC, Groheux D, et al. 18F-FDC PET uptake characterization through texture analysis: investigating the complementary nature of heterogeneity and functional tumor volume in a multi-cancer site patient cohort. J Nucl Med 2015;56:38-44.

[27] Orlhac F, Soussan M, Maisonobe JA, Garcia CA, Vanderlinden B, Buvat I. Tumor texture analysis in 18F-FDG PET: relationships between texture parameters, histogram indices, standardized uptake values, metabolic volumes, and total lesion glycolysis. J Nucl Med 2014;55:414-22.

[28] Hatt M, Visvikis D, Albarghach NM, Tixier F, Pradier O, Cheze-le Rest C. Prognostic value of 18F-FDG PET image-based parameters in oesophagea cancer and impact of tumour delineation methodology. Eur J Nucl Med Mol Imaging 2011;38:1191-202.

[29] Tixier F, Le Rest CC, Hatt M, Albarghach N, Pradier O, Metges JP, et al. Intratumor heterogeneity characterized by textural features on baseline $18 \mathrm{~F}$ FDG PET images predicts response to concomitant radiochemotherapy in esophageal cancer. J Nucl Med 2011;52:369-78.

[30] Yip C, Landau D, Kozarski R, Ganeshan B, Thomas R, Michaelidou A, et al. Primary esophageal cancer: heterogeneity as potential prognostic biomarker in patients treated with definitive chemotherapy and radiation therapy. Radiology 2014;270:141-8.

31] Traverso A, Wee L, Dekker A, Gillies R Repeatability and reproducibility of radiomic features: a systematic review. Int J Radiat Oncol Biol Phys 2018;102:1143-58.

[32] Mackin D, Fave X, Zhang L, Yang J, Jones AK, Ng CS, et al. Harmonizing the pixel size in retrospective computed tomography radiomics studies. PLoS One 2017;12:e0178524.

[33] Zwanenburg A, Leger S, Vallieres M, Lock S. Image biomarker standardisation initiative - feature definitions. 2016 [Accessed March 20th 2017]; Available from: https://arxiv.org/abs/1612.07003v3.

[34] Altman DG, Royston P. What do we mean by validating a prognostic model? Stat Med 2000;19:453-73.

[35] Mallett S, Royston P, Waters R, Dutton S, Altman DG. Reporting performance of prognostic models in cancer: a review. BMC Med 2010;8:21.

[36] Reilly BM, Evans AT. Translating clinical research into clinical practice: impact of using prediction rules to make decisions. Ann Intern Med 2006;144:201-9. 